\title{
Neurological involvement in patients with pulmonary alveolar proteinosis related to MARS mutations
}

\section{Laura Aoust}

Hopital universitaire Necker-Enfants malades

\section{Isabelle Desguerre}

Hopital universitaire Necker-Enfants malades

\section{Nathalie Boddaert}

Hopital universitaire Necker-Enfants malades

Alice Hadchouel ( $\nabla$ alice.hadchouel-duverge@aphp.fr)

Hopital universitaire Necker-Enfants malades

\section{Research}

Keywords: Pulmonary alveolar proteinosis, child, neurological involvement, psychomotor development

Posted Date: July 17th, 2020

DOI: https://doi.org/10.21203/rs.3.rs-41737/v1

License: (9) This work is licensed under a Creative Commons Attribution 4.0 International License. Read Full License 


\section{Abstract}

Background. Pulmonary alveolar proteinosis related to MARS mutations is a rare multisystemic disease with an onset in early infancy. Among extra-respiratory features, neurological involvement was progressively discovered in children. We aimed at describing clinical and radiological neurological features in these patients.

Results. Delay in walking acquisition was noted in $61.5 \%$ and $53.8 \%$ of patients had microcephaly and speech delay. Two lesions were recurrent on brain MRI either isolated or in combination: punctiforme white matter hypersignals and periventricular pseudocysts.

Conclusions. By reporting particular recurrent lesions on brain MRI, we extend the phenotypic spectrum of pulmonary alveolar proteinosis related to MARS mutations. These new data highlight the importance of neurological follow-up and appropriate management in these patients.

\section{Background}

Pulmonary alveolar proteinosis (PAP), first described in 1958 (1), is a rare syndrome characterized by the alveolar accumulation of lipoproteinaceous material (2) and is secondary to abnormalities in surfactant metabolism. A new form of PAP has been described on la Reunion Island, in a cohort of 34 patients (3) with mutations identified in the MARS gene (4). It is characterized by an early onset, associated liver involvement, systemic inflammation, poor prognosis, and frequent progression to lung fibrosis, despite whole-lung lavages that is currently the standard of care in this disorder. The MARS gene, located at locus 13.3 of the long arm of chromosome 12 (12q13.3), encodes the cytosolic methionyl-tRNA synthetase (MetRS), an ubiquitous enzyme responsible for binding the amino acid methionine to its transfer RNA, a necessary step for the initiation of translation and protein synthesis (5). Even if the pathophysiological mechanisms leading from the mutations in MARS to the observed clinical features is still unknown, the fundamental and ubiquitous role of this protein matches with the multisystem phenotype of the patients. We noticed that some children in this cohort had psychomotor delay. The MRIs performed in these patients revealed unusual brain lesions, and given the ubiquitous role of MetRS, theses lesions could represent a characteristic involvement of PAP associated with MARS mutations. Faced with the discovery of these recurrent neuro-radiological abnormalities, the aims of this study were to characterize these abnormalities and correlate it with the medical examination of patients suffering from PAP related to MARS mutations.

\section{Results}

\section{General characteristics of the population}

This study involved 13 patients ( 5 girls and 8 boys). Eight were alive and 5 were dead at the time of data collection. 
The median age at diagnosis was 4.27 months [3.5-8]. These children also had extra-respiratory symptoms, with hepatic dysfunction in 11 of them (84.6\%) with $30.7 \%$ of cirrhosis. Systemic inflammation was found in 5 patients (38.5\%), with $30.8 \%$ of inflammatory anemia. Ten patients $(76.9 \%)$ had experienced a significant malnutrition between 0 and 2 years of age.

\section{Neurological assessment}

\section{a) Development and neurological features}

As shown in Table 1, more than a half of our patients had neurological abnormalities and developmental delay, mainly microcephaly and delayed walking acquisition.

Table 1

Developmental and neurological features

\begin{tabular}{|lll|}
\hline & Number of patients & $\%$ \\
\hline Walking delay & 8 & $61.5 \%$ \\
\hline First words after 18 months & 7 & $53.8 \%$ \\
\hline Microcephaly (HC*<-2DS) & 7 & $53.8 \%$ \\
\hline Oral Disorders & 10 & $76.9 \%$ \\
\hline Neurosensorial abnormalities & 1 & $7.6 \%$ \\
\hline Others anomalies & 1 & $7.6 \%$ \\
\hline Neurological follow-up & 8 & $61.5 \%$ \\
\hline *: head circumference & & \\
\hline
\end{tabular}

This microcephaly was present from birth in 3 children, and persistent in one of them. The other two have gradually regained normal head circumference growth. One patient had acquired microcephaly, increasing during follow-up: his birth head circumference was on a curve of -1.5 SD, with a progressive evolution to -6 SD. His neurological examination was normal. One patient had bilateral perceptive deafness.

\section{b) Educational outcome}

Four children follow a normal school curriculum, three of them without delay and the third repeated two grades.

A patient is enrolled in a school for the hearing impaired, one (currently 5.5 years old with a last available assessment at 4 years old) has never been to school because of his numerous hospitalizations. One patient was dropped out of school at the age of 16 due to disabling anxiety disorders and a fourth was in part-time education. 
At the time of data collection, 3 children were not yet of school age.

\section{c) Brain MRI lesions}

In our series of 13 patients, 9 had brain MRI abnormalities (69.2\%). Two type of lesions were found to be recurrent on our patients: periventricular pseudocysts and hypersignals in white matter. Five patients (38.4\%) had periventricular pseudocysts, 6 (46.1\%) had white matter punctiform sub-cortical or juxta ventricular hypersignals and two patients (15.3\%) had both types of lesions.

- Punctiforme white matter hypersignals

Eight patients had punctiforme white matter hypersignals with variable locations (periventricular in 7 and sub-cortical in 1).

- Periventricular pseudocysts

In our series, these pseudocysts were found on the brain MRIs of 5 children (2 girls and 3 boys). (Fig. 2)

For one of them, this pseudocystic aspect had already been observed on a fetal MRI performed at 34 weeks of menstrual age that was performed because of anomalies detected during obstetrical ultrasound.

\section{d) CT scan}

Brain CT scans were performed in 4 patients (30.7\%) with microcephaly to search for parenchymal calcifications that could evoke congenital CMV infection. No patient had brain calcification.

Differential diagnoses and associated or aggravating factors

\section{a) Neurological or metabolic diseases}

Eight patients (61.5\%) had a metabolic work-up at the time of diagnosis of proteinosis to exclude lysinuric protein intolerance and later for two of them to exclude peroxisomal disease. All the tests were normal.

No neurological diseases were diagnosed in patients in this series, despite investigations in those with significant microcephaly.

\section{b) Factors that may be responsible for abnormalities in psychomotor development}

Data on these factors are presented in Table 2. 
Table 2

Factors that may be responsible for abnormalities in psychomotor development

\begin{tabular}{|c|c|c|c|}
\hline & & $\begin{array}{l}\text { Number of } \\
\text { patients (\%) }\end{array}$ & $\begin{array}{l}\text { Median number } \\
\text { /patient [IQR] }\end{array}$ \\
\hline \multirow[t]{3}{*}{ Neonatal data } & Preterm birth & $2(15.3 \%)$ & \\
\hline & Perinatal anoxia & 0 & \\
\hline & Intrauterine growth delay & $6(46 \%)$ & \\
\hline \multirow{4}{*}{$\begin{array}{l}\text { Extra-pulmonary } \\
\text { involvement }\end{array}$} & Hepatic involvement: & $10(76,9 \%)$ & \\
\hline & Cirrhosis & $4(30,7 \%)$ & \\
\hline & Liver encephalopathy & 0 & \\
\hline & Severe malnutrition & $10(76,9 \%)$ & \\
\hline \multirow[t]{6}{*}{ Hospitalizations } & Neonatal intensive care & $5(38.4 \%)$ & $1[0-1]$ \\
\hline & Pulmonology/General & $12(92,3 \%)$ & $9[4-21]$ \\
\hline & pentatios & $10(76.9 \%)$ & $2[1-4]$ \\
\hline & minemarve cale & $6(46 \%)$ & $1[0-1]$ \\
\hline & rehabilitation & & $14[8-28]$ \\
\hline & TOTAL & & \\
\hline \multirow{3}{*}{$\begin{array}{l}\text { Circulatory failure or } \\
\text { hypoxemia }\end{array}$} & Severe sepsis & $9(69,2 \%)$ & $1[1-3]$ \\
\hline & Acute respiratory failure & $1(7,6 \%)$ & \\
\hline & $\begin{array}{l}\text { Cardiac and/or } \\
\text { respiratory arrest }\end{array}$ & $1(7,6 \%)$ & \\
\hline \multirow[t]{3}{*}{ General anesthesia } & For BAL & $13(100 \%)$ & 26 [25-36] \\
\hline & Other & $9(69,2 \%)$ & $1[1-4]$ \\
\hline & TOTAL & $13(100 \%)$ & $28[26-36]$ \\
\hline
\end{tabular}

We noticed that for a majority of patients, hospitalizations were often long (several weeks to several months), some children never even left the hospital before several months of life.

Severe early malnutrition, often associated with amyotrophy, may explain a delay in motor milestones acquisition.

\section{Discussion}

This study shows the existence of radiological neurological involvement, not yet described, in $58.3 \%$ of our patients suffering from primary alveolar proteinosis due to MARS gene mutations, and characterized 
by two recurrent intra-cerebral lesions: periventricular pseudocysts and white matter hypersignals. In our patients, these isolated or associated lesions do not appear to be related to any other disease.

White matter hypersignals are frequent and non-specific anomalies found mainly on T2 sequences (T2 hyper-signal and hyposignal in T1) and FLAIR. They usually correspond to a process of demyelination or caryotipic anomalies and may be due to hereditary metabolic diseases, or acquired (vascular, infectious as fetal CMV infection, inflammatory, toxic, hypoxic, tumoral and post-traumatic). Their pathogenicity should be correlated with the child's history, age and clinical examination. These hypersignals do not always have a clinical impact and may remain of undetermined origin. Periventricular pseudo-cysts are rare and atypical lesions, which can be seen in pyruvate dehydrogenase and carboxylase deficiencies or in fetal CMV infection.

Some patients also had neurological clinical abnormalities or psychomotor development delay. Microcephaly was the most common clinical neurological feature (50\% of our patients) and a delayed walking acquisition was present in more than half of the patients. The direct relationship between the clinical and radiological neurological abnormalities observed in our series and MARS mutations is difficult to establish for sure. We must consider the factors that play a major role in a child's development, including long and frequent hospitalizations with often prolonged bed rest. Malnutrition in the first two years of life, present in $75 \%$ of our patients, was frequently accompanied by significant amyotrophy, delayed sitting acquisition without help and delayed walking acquisition. However, none of these factors can accounted for microcephaly that was present in more than $50 \%$ of patients in our series, and already at birth for 3 of them.

Given the small number of patients in our series, we were unable to conduct statistical analyses to determine whether the developmental abnormalities in our patients could be secondary to the radiological abnormalities observed or rather correlated to other events that may have influenced their development.

We had no information regarding intelligence quotient, the existence of attention deficit and hyperactivity disorder or autism spectrum disorder, because no child had been assessed by a neuropsychologist. Finally, we did not have any data on the detection of urinary CMV in the neonatal period for these children, to formally rule-out a congenital infection. Dosages of phytanic and pristanic acid, very long chain fatty acids and the search for a deficiency in plasmalogen synthesis were only performed in two patients. Other patients had the standard metabolic check-up at the time of the diagnosis of PAP.

The pathophysiological mechanisms of PAP by mutations of the MARS gene are not yet fully understood. Given the ubiquitous role of MetRS, the lesions observed on brain MRI could represent a characteristic feature of this disease. Arguments in favor of this characteristic neurological impairment are provided by published phenotypic data in patients harboring other mutations in MARS or in genes encoding other aminoacyl tRNA synthetases (ARS). Diseases with mutations in other ARS are responsible for various symptoms in human disease, affecting multiple organs. The first to be described were the mutations of GARS (glycyl-tRNA synthetase), responsible for Charcot-Marie Tooth disease type 2D (6). Since then, other mutations in YARS (tyrosyl tRNA synthetase), AARS (alanyl tRNA synthetase), HARS (histidyl tRNA 
synthetase), KARS (lysyl tRNA synthetase), WARS (tryptophanyl tRNA synthetase) and MARS, were reported in this disease (7). Indeed, MARS mutations, located in the catalytic domain of the enzyme, have been found in two families with type 2 Charcot-Marie Tooth disease (8) (9).

The reason why these different mutations cause specific damage to the peripheral nervous system while ARS are ubiquitous enzymes is not well known. Some authors mention a disruption in protein distribution, with mutations causing a deficit in protein activity, mainly in peripheral neuron axons (which are much longer than axons in the central nervous system). For GARS, the pathophysiological hypothesis is that the mutation would modify proteins and global protein content, which would directly antagonize a signaling pathway essential to motor neurons' survival. The hypothesis of mitochondrial dysfunction in GARS mutants is also raised, leading to dendritic morphological abnormalities (7). Other data have also shown synaptic maturation abnormalities with progressive and specific degeneration of the neuromuscular junction in Drosophila with a GARS mutation (10). In our patients, that bear the recurrent double mutation Ser567Leu/Ala393Thr in MARS, such involvement of the peripheral nervous system was never described.

A recently published study (11) by Fuchs et al. identified 112 patients (107 in the literature and 5 new cases) with a phenotype characterized by multi-organ involvement secondary to mutations in cytosolic AARS, DARS (aspartyl-RNAt synthase), GARS, HARS, IARS (isoleucyl-ARNt synthetase), KARS, LARS (leucylARNt synthetase), MARS, RARS (arginyl-ARNt synthetase), SARS (seryl-ARNt synthetase), VARS (valylARNt synthetase), YARS, and QARS (glutaminyl-ARNt synthase). Common features were: damage to the central ( $100 \%$ of cases) or peripheral nervous system, pulmonary interstitial disease, gastrointestinal disease with growth delay, hepatic, renal and hematological involvements with anemia.

Among the 107 cases already published, the central neurological disorders described were: psychomotor retardation, microcephaly, hypotonia, seizures, encephalopathy, spasticity, ataxia and agitation. Regarding patients with MARS mutations, the authors reported patients from our initial series $(3,4)$ for whom the neurological have not yet been published, and also 2 case-reports with one patient (12) and a Chinese sibling of 2 boys (13). These patients had a pulmonary and hepatic phenotype compatible with that of Reunion Island's PAP. Neurologically, these two studies report delayed motor development with hypotonia in all 3 patients. Only the two boys from the same sibling had brain MRI abnormalities: an arachnoid cyst for the first, and bilateral ventricular enlargement for the second.

Fuchs et al. also report 5 new cases of patients with IARS mutations (in two patients), KARS, LARS and QARS respectively and describe a microcephaly in three of them (IARS mutation and QARS) with severe developmental delay. One of them also had a hearing loss treated with a cochlear implant, as a patient in our series. Brain MRI abnormalities were found in 3 out of 5 patients: white matter hypersignals associated with brain atrophy in one patient, niger locus hypersignals in another, and delayed myelination in the 3rd patient. Among those patients, three (with the IARS and YARS mutation) had PAP.

For all these diseases, the pathophysiological mechanism evoked by the authors is a decrease in enzymatic activity, affecting more particularly organs sensitive to amino acid depletion (liver brain, lung and muscles) or organs with rapid cell renewal such as the intestine. 
Three other case-reports were published since the review of Fuchs et al. and reported patients with other mutations in the MARS gene. Rips et al. (14) reported a boy, born from non-related parents, who developed neonatal anemia, liver failure, hypothyroidism and then respiratory distress at the age of 4 months, leading to the diagnosis of interstitial lung disease. As our patients, he also had poor weight growth, delayed psychomotor development and hypotonia, but his brain MRI was normal. Abuduxikuer et al. (15) described the case of a 5-month-old girl, with interstitial lung disease, failure to thrive, developmental delay, hepatomegaly with severe steatosis, anemia, and thrombocytosis. This patient died of respiratory failure at the age of 11 months.

Finally, Alzaid et al (16) reported a 6-month-old boy with interstitial lung disease compatible with PAP, intermittent fever, axial hypotonia, hepatomegaly, hypoglycemia, hypothyroidism, anemia, thrombocytopenia. This patient also died of respiratory failure. Despite their developmental delay, these two patients did not undergo brain imaging. Overall, these 3 new cases with MARS mutations presented psychomotor development anomalies, like our patients carrying the double mutation Ser567Leu / Ala393Thr. However, these case-reports do not describe neuro-radiological abnormalities.

Finally, brain MRI abnormalities isolated in our patients are distinct from those identified in patients harboring mutations in genes encoding mitochondrial ARS. Indeed, those mutations are responsible for deep developmental disorders of myelination leading to often severe leukodystrophies (17).

Thus, disorders secondary to mutations in genes encoding aminoacyl-tRNA synthetases seem to have common clinical characteristics, with almost constant neurological and pulmonary damage. Neurological damage secondary to MARS mutations had so far only been described in a few cases of Charcot-Marie Tooth disease, which is a disease of the peripheral nervous system, and in 5 cases of children with MARS mutations but without brain MRI-specific abnormalities. Our study provides strong arguments for the existence of characteristic central neurological impairment in patients with the double mutation Ser567Leu/Ala393Thr of the MARS gene associated with the PAP phenotype in these patients.

\section{Conclusions}

By a retrospective analysis of patients bearing the double recurrent Reunion Ser567Leu/Ala393Thr mutations in MARS, we extend the phenotypic spectrum of this multi-organ systemic disease and add a cerebral involvement to the already known pulmonary, hepatic and inflammatory features. These new data highlight the importance of neurological follow-up and appropriate management in these patients.

\section{Methods}

\section{Study design}

This is a retrospective, monocentric cohort study. All patients with PAP and double Reunion Island mutations in the MARS gene (Ser567Leu/Ala393Thr) followed at Necker-Enfants Malades Hospital, still alive or deceased and having had at least one cerebral MRI during their follow-up were included. 
This study was approved by the Committee for the Evaluation of Observational Research Protocols (CEPRO) of the Societé de Pneumologie de Langue Francaise (SPLF) on May 3, 2018 (nCEPRO 2018006).

\section{Data collection}

The data collection was conducted in patients' medical records using a standardized grid and focused on neurological examination and psychomotor developmental data. The following items were retrieved:

- Age of walking acquisition: delay was defined by walking not achieved by 18 months

- Age of first words : language delay was defined by no words by the age of 2 years and inability to talk in short sentences by the age of 3 years

- Microcephaly defined by a head circumference measured below - 2 standard deviations (SD)

- Oral disorders

- Neurosensorial abnormalities (deafness, blindness), neurological abnormalities

- Need for neurological follow-up

- Academic delay

Radiological data were collected from brain MRI but also from brain CT scan or transfontanellar ultrasound. All images were read by the same radiologist.

We also looked for all factors that may have influenced the neurological development of these children: gestational age and birth conditions, neurological family history, the number of hospitalizations (including intensive care hospitalizations), the severity of respiratory impairment assessed by the need for oxygen therapy or non-invasive ventilation, episodes of low brain flow and general anesthesia.

Finally, we looked for the results of biological examinations allowing us to exclude differential diagnoses that could also lead to neurological damage, including chromatography of amino acids from blood and urine, dosage of phytanic and pristanic acid, very long chain fatty acids, and the search for a deficit in the synthesis of plasmalogens, to rule-out peroxisomal disorders, and cytomegalovirus (CMV) PCR in blood, BAL and urines when those data were available, to rule-out a neonatal infection.

\section{Statistical analyses}

Non continuous variables were expressed as a percentage on the number of patients for whom the information was available and continuous variables were expressed as a median and their inter-quartile range (IQR).

\section{Declarations}

Ethics approval and consent to participate: this study was approved by the Committee for the Evaluation of Observational Research Protocols (CEPRO) of the Société de Pneumologie de Langue Francaise (SPLF) on May 3, 2018 ( ${ }^{\circ}$ CEPRO 2018-006). As it was a retrospective study by collecting data from 
medical files, parental consent was not required but parents are informed that, as their child is followed in university hospital, data from his medical file may be used for research purpose.

Consent for publication: As it was a retrospective study by collecting data from medical files, parental consent was not required but parents are informed that, as their child is followed in university hospital, data from his medical file may be used for research purpose.

Availability of data and materials: data were collected form medical files and stored in an anonymous database.

Funding: not applicable

The authors have no competing interests

Authors' contributions: LA, ID, NB and AH made substantial contributions to the conception and design of the work. LA collected the data. LA, ID, NB and AH contribute to the analysis and interpretation of data. LA and $A H$ have drafted the work and $L A, I D, N B$ and $A H$ substantively revised it. LA, ID, NB and $A H$ have approved the submitted version. LA, ID, NB and AH have agreed both to be personally accountable for the author's own contributions and to ensure that questions related to the accuracy or integrity of any part of the work, even ones in which the author was not personally involved, are appropriately investigated, resolved, and the resolution documented in the literature.

Acknowledgements: We thank Dr Laurent Enaud who has been involved for a long time in the care of numerous families with this disease in Réunion Island.

\section{References}

1. Rosen SH, Castleman B, Liebow AA. Pulmonary alveolar proteinosis. N Engl J Med. 5 juin 1958;258(23):1123-42.

2. de Blic J. Pulmonary alveolar proteinosis in children. Paediatr Respir Rev. déc 2004;5(4):316-22.

3. Enaud L, Hadchouel A, Coulomb A, Berteloot L, Lacaille F, Boccon-Gibod L, et al. Pulmonary alveolar proteinosis in children on La Réunion Island: a new inherited disorder? Orphanet J Rare Dis. 14 juin 2014;9:85.

4. Hadchouel A, Wieland T, Griese M, Baruffini E, Lorenz-Depiereux B, Enaud L, et al. Biallelic Mutations of Methionyl-tRNA Synthetase Cause a Specific Type of Pulmonary Alveolar Proteinosis Prevalent on Réunion Island. Am J Hum Genet. 7 mai 2015;96(5):826-31.

5. Kaminska M, Havrylenko S, Decottignies P, Gillet S, Le Maréchal P, Negrutskii B, et al. Dissection of the structural organization of the aminoacyl-tRNA synthetase complex. J Biol Chem. 6 mars 
2009;284(10):6053-60.

6. Antonellis A, Ellsworth RE, Sambuughin N, Puls I, Abel A, Lee-Lin S-Q, et al. Glycyl tRNA synthetase mutations in Charcot-Marie-Tooth disease type 2D and distal spinal muscular atrophy type $\mathrm{V}$. Am J Hum Genet. mai 2003;72(5):1293-9.

7. Boczonadi V, Jennings MJ, Horvath R. The role of tRNA synthetases in neurological and neuromuscular disorders. FEBS Lett. mars 2018;592(5):703-17.

8. Gonzalez M, McLaughlin H, Houlden H, Guo M, Yo-Tsen L, Hadjivassilious M, et al. Exome sequencing identifies a significant variant in methionyl-tRNA synthetase (MARS) in a family with late-onset CMT2. J Neurol Neurosurg Psychiatry. nov 2013;84(11):1247-9.

9. Hyun YS, Park HJ, Heo S-H, Yoon BR, Nam SH, Kim S-B, et al. Rare variants in methionyl- and tyrosyltRNA synthetase genes in late-onset autosomal dominant Charcot-Marie-Tooth neuropathy. Clin Genet. déc 2014;86(6):592-4.

10. Grice SJ, Sleigh JN, Motley WW, Liu J-L, Burgess RW, Talbot K, et al. Dominant, toxic gain-of-function mutations in gars lead to non-cell autonomous neuropathology. Hum Mol Genet. 1 août 2015;24(15):4397-406.

11. Fuchs SA, Schene IF, Kok G, Jansen JM, Nikkels PGJ, van Gassen KLI, et al. Aminoacyl-tRNA synthetase deficiencies in search of common themes. Genet Med. 6 juin 2018;

12. van Meel E, Wegner DJ, Cliften P, Willing MC, White FV, Kornfeld S, et al. Rare recessive loss-offunction methionyl-tRNA synthetase mutations presenting as a multi-organ phenotype. BMC Med Genet. 8 oct 2013;14:106.

13. Sun $Y, H u$ G, Luo J, Fang D, Yu Y, Wang $X$, et al. Mutations in methionyl-tRNA synthetase gene in a Chinese family with interstitial lung and liver disease, postnatal growth failure and anemia. J Hum Genet. juin 2017;62(6):647-51.

14. Rips J, Meyer-Schuman R, Breuer O, Tsabari R, Shaag A, Revel-Vilk S, et al. MARS variant associated with both recessive interstitial lung and liver disease and dominant Charcot-Marie-Tooth disease. Eur J Med Genet. 12 avr 2018;

15. Abuduxikuer K, Feng J-Y, Lu Y, Xie X-B, Chen L, Wang J-S. Novel methionyl-tRNA synthetase gene variants/phenotypes in interstitial lung and liver disease: A case report and review of literature. World J Gastroenterol. 28 sept 2018;24(36):4208-16.

16. Alzaid M, Alshamrani A, Harbi ASA, Alenzi A, Mohamed S. Methionyl-tRNA synthetase novel mutation causes pulmonary alveolar proteinosis. Saudi Med J. févr 2019;40(2):195-8.

17. Fine AS, Nemeth CL, Kaufman ML, Fatemi A. Mitochondrial aminoacyl-tRNA synthetase disorders: an emerging group of developmental disorders of myelination. Journal of Neurodevelopmental Disorders [Internet]. déc 2019 [cité 12 juill 2020];11(1). Disponible sur: https://jneurodevdisorders.biomedcentral.com/articles/10.1186/s11689-019-9292-y

\section{Figures}




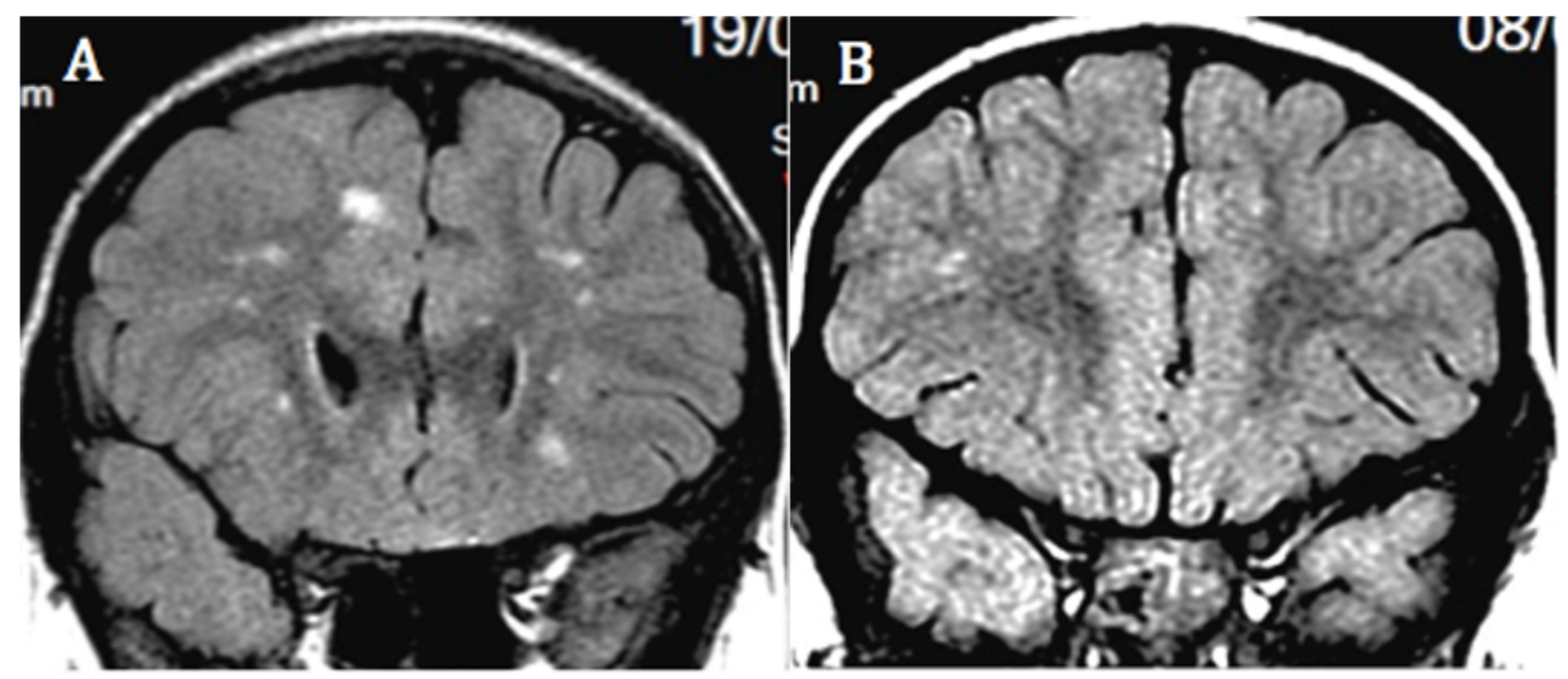

Figure 1

Coronal sections of brain MRI, FLAIR sequence A: 9 years and 10 months old patient with PAP diagnosed at 1 year old B: 3 years and 9 months old patient with PAP diagnosed at 2 months old These images show subcortical white matter hypersignals.

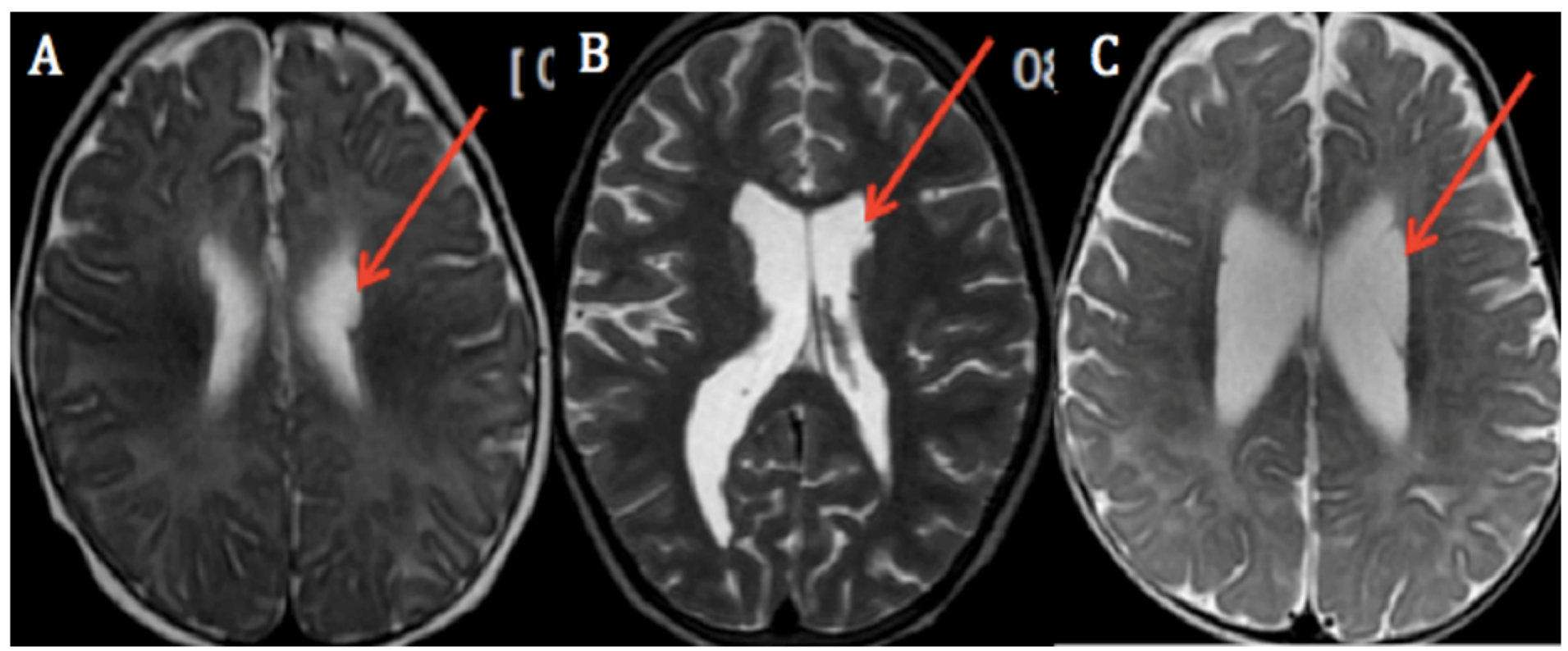

Figure 2

Axial section of brain MRI, T2 sequence. A: 5-month-old patient with PAP diagnosed at 4 months old. B: 11 years and 3 months old patient with PAP diagnosed at 5.5 months old. C: 5.5 months old patient with PAP diagnosed at 4.5 months of age. These images show periventricular pseudocysts indicated by red arrows 